\title{
Patterns and predictors of recurrence after open radical cystectomy for bladder cancer: a comprehensive review of the literature
}

\author{
Andrea Mari ${ }^{1,2} \cdot$ Riccardo Campi $^{1} \cdot$ Riccardo Tellini $^{1} \cdot$ Giorgio Gandaglia $^{3} \cdot$ Simone Albisinni ${ }^{4}$. \\ Mohammad Abufaraj ${ }^{2,5}$. Georgios Hatzichristodoulou ${ }^{6}$. Francesco Montorsi ${ }^{3} \cdot$ Roland van Velthoven $^{4}$. \\ Marco Carini ${ }^{1}$ - Andrea Minervini ${ }^{1}$ - Shahrokh F. Shariat ${ }^{2,7,8,9,10}$
}

Received: 14 August 2017 / Accepted: 27 October 2017 / Published online: 16 November 2017

(c) The Author(s) 2017. This article is an open access publication

\begin{abstract}
Purpose To review the currently available literature reporting the patterns of recurrence and their predictive factors after open radical cystectomy (RC) for bladder cancer.

Methods A review of the literature was performed using the MEDLINE, Scopus and Web of Sciences databases from January 1997 to May 2017. The PRISMA guidelines were followed for the conduct of the study.

Results Local recurrence rate ranges between 30 and 54\%. Distant recurrence is not often standardized and is reported in up to $50 \%$ of cases. The overall 5-year recurrence-free survival rates from 58 to $81 \%$. The mean follow-up of studies included in the analysis ranged from 18 to 350 months. Details on the most important demographic and epidemiological, clinical, histologic and pathologic predictors of recurrence after radical cystectomy are provided through an evidence-based approach. The impact of the extension of lymph node dissection on recurrence after RC is investigated.

Conclusions A correct prognostic assessment is essential for patients undergoing radical cystectomy for bladder cancer, thereby potentially improving their oncologic outcomes.
\end{abstract}

Keywords Bladder cancer $\cdot$ Recurrence $\cdot$ Radical cystectomy $\cdot$ Lymph node dissection $\cdot$ Neoadjuvant chemotherapy · Adjuvant chemotherapy

\section{Introduction}

Bladder cancer (BC) is the 9th most commonly diagnosed cancer and is ranked 13 th for cancer deaths in the overall

Electronic supplementary material The online version of this article (https://doi.org/10.1007/s00345-017-2115-4) contains supplementary material, which is available to authorized users.

Shahrokh F. Shariat

shahrokh.shariat@meduniwien.ac.at

1 Department of Urology, University of Florence, Careggi Hospital, Florence, Italy

2 Department of Urology, Medical University of Vienna, Vienna, Austria

3 Division of Oncology/Unit of Urology, IRCCS San Raffaele Hospital, URI, Milan, Italy

4 Department of Urology, Institut Jules Bordet, Université Libre de Bruxelles, Bruxelles, Belgium

5 Division of Urology, Department of Special Surgery, Jordan University Hospital, The University of Jordan, Amman, Jordan population in 2015 worldwide. Approximately $30 \%$ of newly diagnosed bladder cancer patients present with muscle-invasive BC (MIBC) disease with $10-15 \%$ of cases already metastatic at diagnosis. The risk of progression for high-risk non-MIBC after 5 years is $45 \%$ [1]. The standard treatment for patients with clinically localized

6 Department of Urology and Pediatric Urology, Julius-Maximilians-University of Würzburg, Würzburg, Germany

7 Karl Landsteiner Institute of Urology and Andrology, Vienna, Austria

8 Department of Urology, University of Texas Southwestern Medical Center, Dallas, TX, USA

9 Department of Urology, Weill Cornell Medical College, New York, NY, USA

10 Department of Urology and Comprehensive Cancer Center, Vienna General Hospital, Medical University of Vienna, Währinger Gürtel 18-20, 1090 Vienna, Austria 
(cT2-T4a N0M0) MIBC is radical cystectomy (RC) with bilateral pelvic lymph node dissection (PLND). The use of neoadjuvant chemotherapy (NAC) may be a valid option for MIBC but its benefit is still debated. In addition, it is reasonable to propose immediate $\mathrm{RC}$ to those patients with non-MIBC at highest risk of progression. Furthermore, early RC should be offered to all patients with non-MIBC failing endovesical therapy [1].

Unfortunately, a significant proportion of patients with MIBC experiences recurrence and eventually death after $\mathrm{RC}$. There is no definitive evidence regarding the recurrence rate after RC. In particular, the definition of local and distant recurrence is not standardized, the recurrencefree survival (RFS) is highly variable and the specific timing of recurrence is not well defined across the published series [2-5]. Several factors have been variously associated with recurrence after RC. However, the different patient selection and the variable use of preoperative treatments among studies could affect the impact on the analysis of predictors of recurrence after RC.

Under this light, we aimed to review the currently available literature reporting the patterns of recurrence and their predictive factors after open RC. Moreover, we critically assessed the current limitations of the published series outlining potential implications for future research.

\section{Materials and methods}

A comprehensive review of the English-language literature was performed using the MEDLINE, Scopus and Web of Sciences databases from January 1st, 1997 to May 1st, 2017 following the preferred reporting items for systematic reviews and meta-analyses (PRISMA) statement using the following keywords: "recurrence" or "relapse" or "metastasis" and "bladder cancer" or "urothelial bladder cancer" and "radical cystectomy" (Fig. 1). After a first screening based on study title and abstract, all papers were assessed based on full text and excluded with reasons when appropriate. Two reviewers (A.M. and R.C.) carried out this process independently. Disagreement was solved by a third party (G.H.). The list of articles that were judged to be highly relevant by these authors was reviewed by all coauthors until a final consensus was reached on the articles included in the analysis. The "riskof-bias" evaluation of the studies included to investigate the recurrence rate $(n=48)$ was assessed according to the Cochrane Handbook for Systematic Reviews of Interventions [6].

\section{Evidence synthesis}

A total of 1481 articles were identified. After exclusion of duplicates, case reports, congress abstracts, editorials and papers with topics that were not specific for this review, 1125 papers were selected for full-text assessment of eligibility. After full-text evaluation, 355 studies were included for the analysis of predictors of recurrence in patients treated with radical cystectomy. Of these, 48 studies were included for the assessment of recurrence rate (Fig. 1).

\section{Type of studies and quality of the evidence}

For the analysis of the predictors of recurrence, 313 studies were retrospective, 14 were prospective and 27 were randomized clinical trials (RCTs). Sample size ranged between 33 and 9064 patients. Most series involved multiple surgeons. For the analysis of recurrence rate, 29 studies were retrospective, 3 were prospective and 16 were RCTs. Sample size ranged between 110 and 9064 patients. Risk of bias evaluation is summarized in Supplementary Table 1.

\section{Definition and controversies}

EAU Guidelines defined local recurrence after RC as any recurrence that takes place in soft tissues at the original surgical site or lymph nodes (LN) in the area of PLND [1]. Data in literature are very inconsistent, as a detailed discrimination between local and distant recurrence is not always present. Similarly, the percentages of RFS are highly variable even across large surgical series. Moreover, some studies include different neoadjuvant or adjuvant chemotherapy (AC) regimens, which may hinder the overall interpretation of surgical series.

\section{Recurrence rates in patients treated with open RC}

In a retrospective study involving 4118 patients naïve of NAC treated at several institutions, the RFS was 63, 60 and $57 \%$ at 3, 5 and 10 years, respectively [2]. Xylinas et al. [3] analyzed the oncologic outcomes of 2145 patients naïve of NAC treated at European and US institutions with open RC for pT1-T3N0 urothelial BC. The RFS was $68 \%$ at a median follow-up of 48 months. Similarly, Zehnder et al. reported the oncologic outcomes of 1488 patients at a median followup of 162 months [4]. Overall, 6\% received NAC and 3\% preoperative radiotherapy (RT). The 5- and 10-year RFS were 68 and 64\%, respectively, with a time to recurrence ranged from 0 to 29.2 years.

The recurrence rates in patients treated with NAC and open RC are still undetermined. In most of the retrospective studies, a low percentage of patients receive NAC 


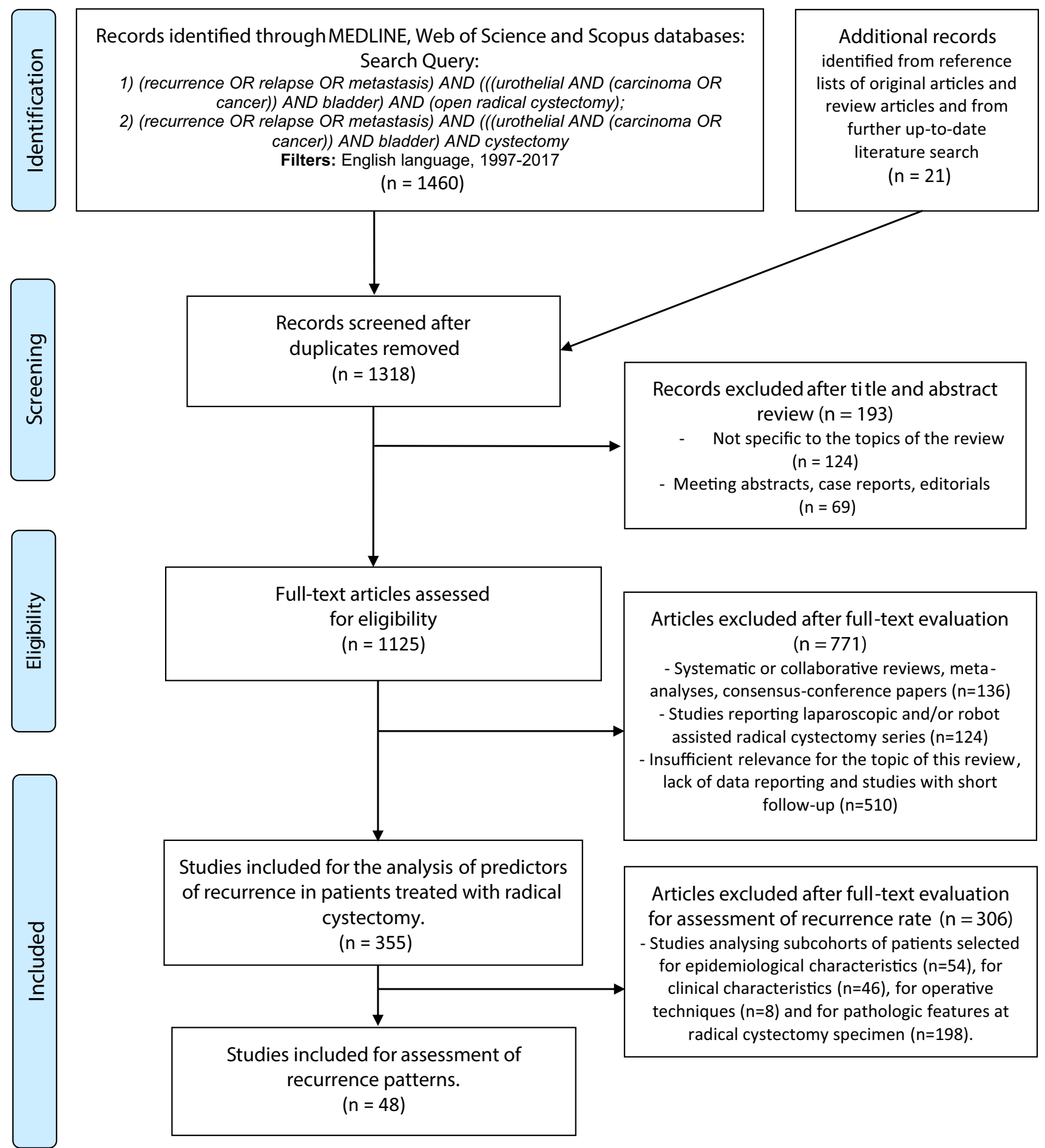

Fig. 1 Flow chart for article selection process

due to the contraindications and the underuse reported in several centers. The best shreds of evidence come from RCTs, even if the real number of patients effectively submitted to the combined therapy is difficult to estimate due to the variable number of patients declining NAC and of those not completing the full number of cycles for adverse events.

In the experimental arm from the BA06 30894 (International Collaboration of Trialists) RCT, $491 \mathrm{BC}$ patients with T2-4N0-xM0 stage underwent NAC (cisplatin, methotrexate, and vinblastine) and radical treatment (50\% RC, 
$42 \%$ radical $\mathrm{RT}$ and $8 \%$ preoperative $\mathrm{RT}$ plus $\mathrm{RC}$ ) [7]. The median locoregional disease-free and metastasis-free survival for the group were 23 months (47\% at 3 years) and 32 months (45\% at 3 years). In the Southwest Oncology Group (SWOG) RCT, 153 patients with T2-4N0M0 stage $\mathrm{BC}$ were randomly assigned to methotrexate, vinblastine, doxorubicin, and cisplatin (MVAC) NAC and subsequent RC [8]. At 5 years, $57 \%$ of the patients were alive. After a median follow-up of 8.7 years, $35 \%$ of patients died of disease. In a meta-analysis, the oncologic outcomes of 2491 patients treated with NAC and radical treatment (RC, radical RT, or preoperative RT and RC) from 10 clinical RCTs were reported. The locoregional disease-free survival was $166 / 217(76.5 \%)$ and 1232/1963 (62.8\%) in patients treated with single agent platinum and platinum-based combination, respectively. The metastasis-free survival rate was $154 / 217(71 \%)$ and $1181 / 1963(60.2 \%)$ in patients treated with single agent platinum and platinum-based combination, respectively.

According to the studies included in this review, the reported local recurrence rate after open RC was between 29.9 and $53.8 \%$, and the 5-year RFS rates from 58 to $80.9 \%$. The median follow-up of studies included in the analysis ranged from 18 to 350 months.

\section{Predictive factors of recurrence-free survival in patients treated with open $\mathrm{RC}$}

\section{Demographic and epidemiologic characteristics}

Although men are three to four times more likely to develop BC, several epidemiologic reports associated female gender with a more advanced disease and worse survival rates [9, 10]. Messer et al. [11] confirmed that female gender, after adjusting to standard clinical and pathologic features, was associated with an increased risk for cancer-specific mortality (CSM) and disease recurrence compared to male gender in patients undergoing open RC. In other studies, gender was not associated with outcomes in stage-adjusted analyses, whereas pathologic tumor stage remained the most powerful factor influencing the course of disease in both genders [12].

Elderly patients have more years to compound comorbidities. As such, they are associated with higher mortality after RC [13]. In a retrospective analysis of 605 patients treated with open RC, Horovitz et al. reported that octogenarians had comparable RFS to the younger counterparts [14]. Conversely, an overview of the Bladder Cancer Research Consortium reported that patients aged $>80$ years had a significantly greater risk of disease recurrence than patients aged $\leq 60$ years [15]. However, the retrospective nature of the studies suffered of the selection bias related to the higher use of bladder-sparing techniques in older patients and to the lower use of both NAC and AC.
In a retrospective multicentre study on more than 1500 patients, current smoking status and high cumulative smoking exposure at the time of $\mathrm{RC}$ was significantly associated with advanced tumor stage, nodal metastasis, disease recurrence, and CSM in patients treated with RC for high-risk non-MIBC and MIBC [16]. Smoking cessation > 10 years prior to RC mitigates the detrimental effect of smoking. Conversely, in two different studies, smoking was not an independent predictor of RFS $[17,18]$. In addition, smoking seemed to affect survival only among men, but not in women, who had overall a poorer survival compared to the male gender [17]. The most important studies of this paragraph are summarized in Table 1.

\section{Clinical characteristics}

Several serological parameters have been investigated as possible predictors of recurrence in patients treated with open RC for MIBC. The pre-treatment neutrophil-to-lymphocyte ratio (NLR) is an emerging marker of host inflammation. Several studies reported the impact of NLR on oncologic outcomes in patients undergoing RC. The threshold varied among studies (from 2, 7 to 3 ). NLR was significantly associated with extravesical tumor extension, node involvement and was an independent predictor of relapse and CSM after RC [19, 20]. Similarly, the lymphocyte-to-monocyte ratio (LMR), an alternative marker of inflammation, was significantly associated with RFS [19, 21]. Preoperative hypoalbuminemia $(<3.5 \mathrm{~g} / \mathrm{dL})$ [22] and low albumin/globulin ratio $(\geq 1.60)[23]$ were found to be independent predictors of RFS and OS, and RFS and cancer-specific survival (CSS), respectively. Moreover, the low albumin/globulin ratio remains an independent predictor of RFS and CSS even in a subcohort of patients with normal serum albumin [23].

In a retrospective analysis of 906 treated with RC, thrombocytosis (defined as $>400,000$ platelets $/ \mathrm{mcl}$ ) was significantly associated with adverse pathologic disease stage and LN involvement [24]. Thrombocytosis was independently associated with OS, but not with RFS, after adjusting for clinicopathological factors.

A recent meta-analysis analyzed 11 and 7 studies evaluating the impact of anemia and hemoglobin level as continuous variable, respectively, in patients treated with RC. Both anemia and hemoglobin level were independently associated with increased all-cause mortality, CSM and disease recurrence [25].

The AB0 blood group antigen was found to be associated with a higher tumor stage, but not a higher risk of relapse in patients treated with $\mathrm{RC}$ [26]. In a subanalysis of patients treated with $\mathrm{AC}$ after $\mathrm{RC}$, the $\mathrm{AB}$ blood group antigen expression was significantly associated with a worse CSM compared to the $\mathrm{A} 0, \mathrm{~B} 0$ and 00 blood groups [26].

The current evidence supports the prognostic role of inflammatory blood-based markers and physical 


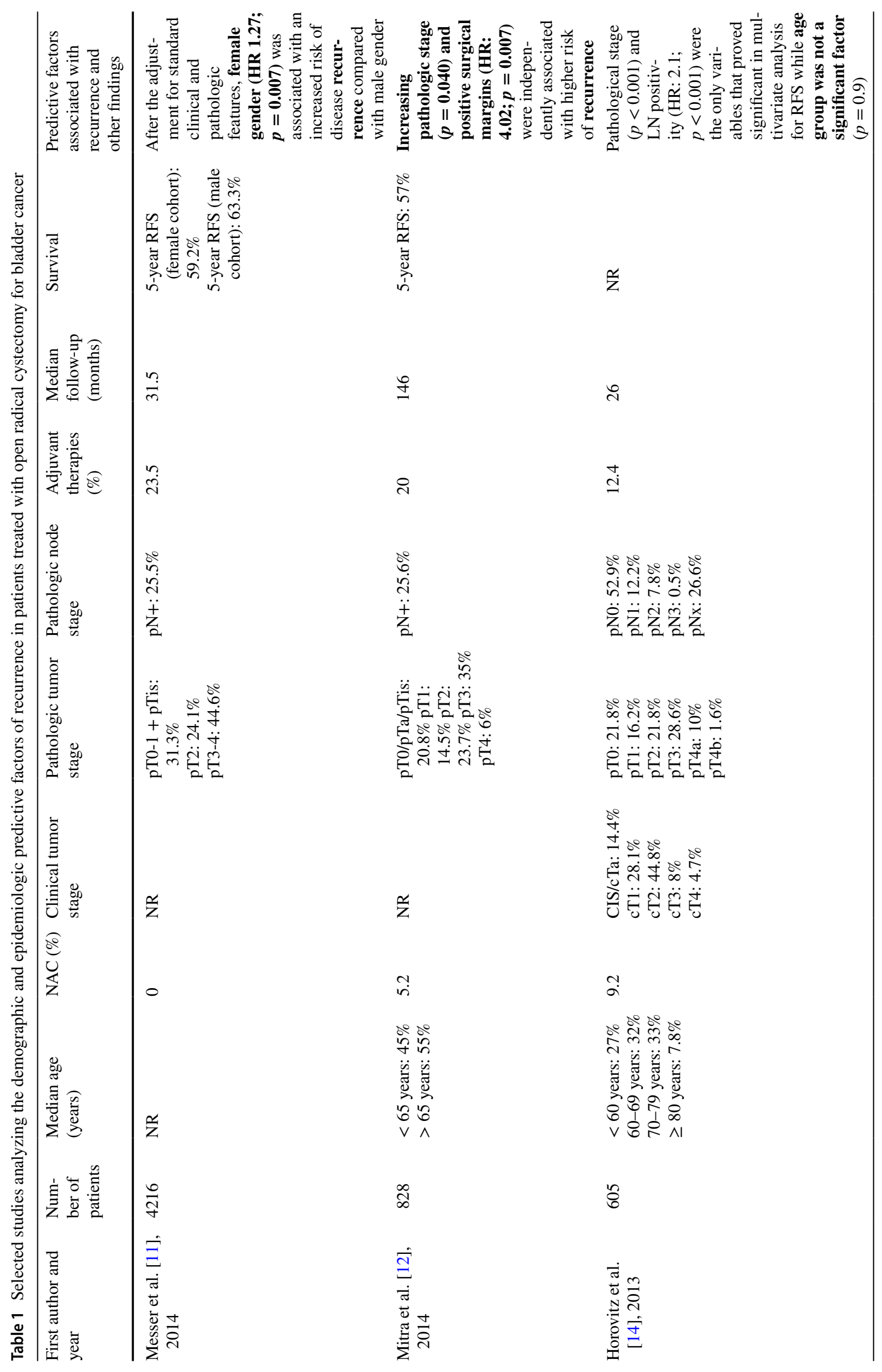




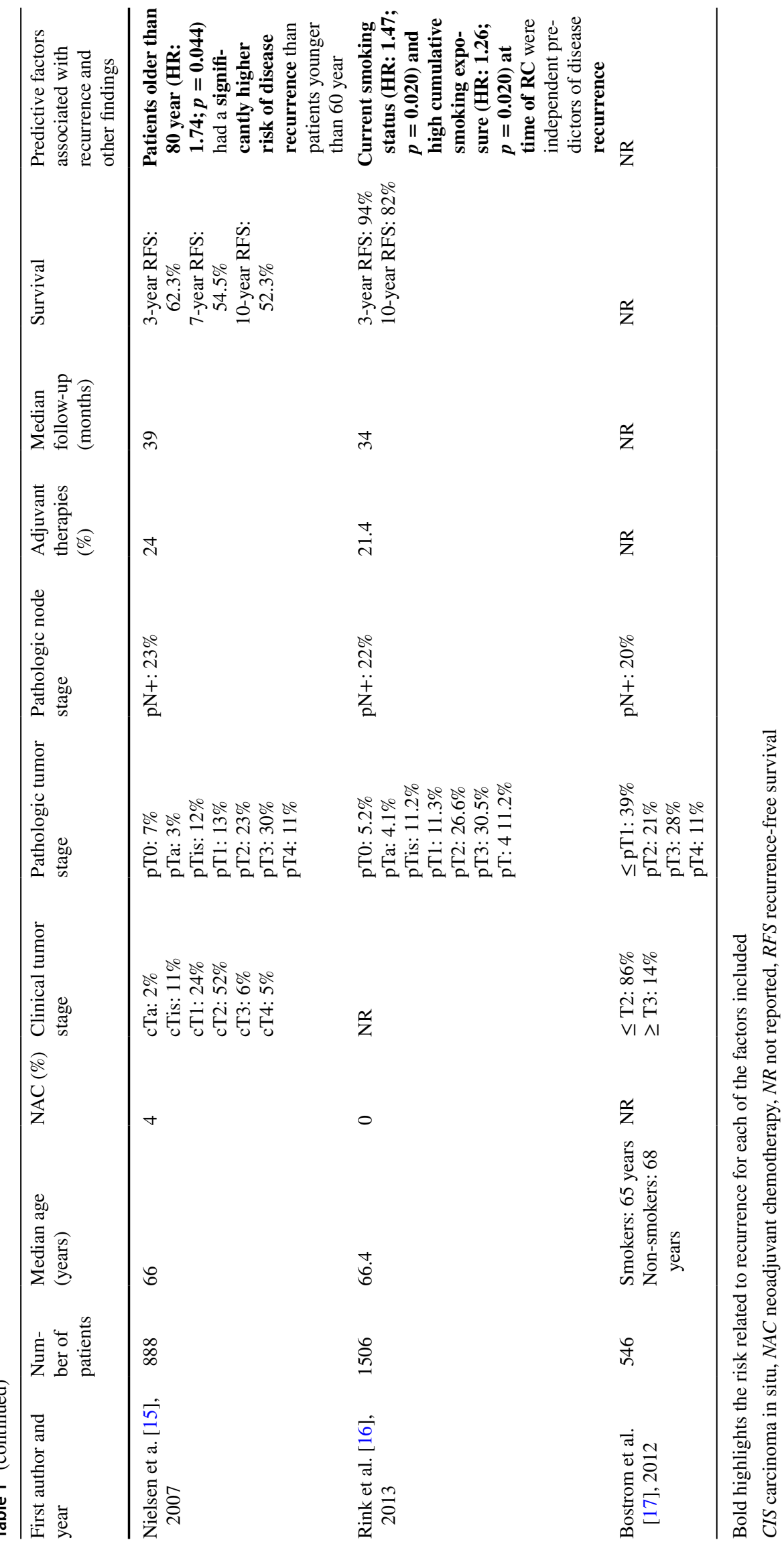


characteristics on oncologic outcomes after RC. Indeed, systemic inflammation seems to play a critical role in the pathogenesis of malnutrition. In a restricted cohort of 117 Japanese patients, the prognostic nutritional index (PNI) but not the controlling nutritional status (CONUT) was significantly associated with disease-specific survival after RC [27]. However, further studies are necessary to demonstrate the association between the preoperative nutritional status and oncologic outcomes in patients treated with RC for BC.

Patients with comorbidities are often less likely to have PLND and NAC [28]. Several studies reported that a higher comorbidity score was significantly associated with lower OS, but not RFS in patients undergoing open RC [29, 30]. However, after developing a recurrence, the presence of comorbidities was shown to have an independent correlation with lower survival [30]. A retrospective study including 4118 patients treated with open RC showed that obesity was significantly associated with higher tumor grade, soft-tissue surgical margins (STSMs) and worse oncologic outcomes [2]. Other authors reported that a body mass index $>30$ is an independent predictor of disease recurrence and CSM [31].

In patients with diabetes mellitus undergoing RC, diabetes mellitus was significantly associated with worse CSS [32]. Moreover, metformin use was related to a decreased risk of disease recurrence and CSM [32].

Clinical staging at transurethral resection (TUR) is a fundamental diagnostic and therapeutic tool for patients with BC $[1,33]$. However, the clinical stage at TUR was shown to be divergent from pathologic tumor stage in more than a half of the reported cases [34]. Patients with clinical understaging were associated with a higher risk of $\mathrm{LN}$ involvement at RC specimen and with worse CSM after RC [34].

In a retrospective evaluation of 297 patients treated with open RC without NAC, Culp et al. identified the presence of micropapillary or neuroendocrine features together with lymphovascular invasion (LVI) at TUR specimen, hydroureteronephrosis and cT3b-T4a disease as readily assessable clinical factors to define a high-risk disease [35]. Patients with high-risk disease were found to have a higher probability of upstaging at RC specimen and to have a decreased diseasespecific and progression-free survival probabilities compared to low-risk patients. These results have been recently validated in an external cohort [36]. Indeed, this clinical preoperative risk grouping might help the decision-making process regarding the administration of NAC $[35,36]$. The most important studies of this paragraph are summarized in Table 2.

\section{Histologic patterns}

The prognostic value of pathologic features on recurrence is of great importance in patients treated with open RC. Lotan et al. [37] reported that LVI was an independent predictor of local, distant and overall recurrence in $\mathrm{pN} 0$ patients treated with open RC. Several studies unanimously confirmed that LVI at RC specimen has an independent association with RFS after surgery. In a report of 2000 patients, variant histology $(\mathrm{VH})$ of urothelial $\mathrm{BC}$ at bladder specimen accounts for approximately $25 \%$ of cases of patients undergoing $\mathrm{RC}$ [38]. Patients with $\mathrm{VH}$ had a significantly higher disease recurrence rate compared to those with pure urothelial BC patients or with squamous-cell differentiated BC. Nonetheless, this effect did not remain significant on the multivariable model [38]. Soave et al. [39] demonstrated that the presence of $\mathrm{VH}$ and non-squamous cell differentiation had not a significant impact on oncological outcomes. Similarly, Fairey et al. demonstrated that micropapillary variant of urothelial BC had similar oncological outcomes after open $\mathrm{RC}$ to those with pure urothelial $\mathrm{BC}$ after controlling for relevant clinical and pathologic factors [40]. Recently, Moschini et al. reported that pure $\mathrm{VH}$, mostly represented by small-cell and micropapillary $\mathrm{VH}$, compared to the pure urothelial $\mathrm{BC}$ at $\mathrm{RC}$ specimen was an independent predictor of CSM and RFS [41]. In a cohort of patients with cT1-4N0-2 treated with cisplatin-based NAC and RC, the squamous or glandular $\mathrm{VH}$ was an independent predictor of pathologic downstaging [42]. Furthermore, these patients presenting squamous or glandular VH had comparable overall survival compared to those with pure urothelial BC.

Contrasting results were reported regarding the impact of carcinoma in situ (CIS) at RC specimen on recurrence-free survival. In a retrospective evaluation, the clinical data of 812 patients treated with RC were analyzed [43, 44]. The presence of concomitant CIS ( $46 \%$ of cases) was significantly associated with urethra involvement at the pathologic specimen and, in patients with organ-confined disease, was independently associated with disease recurrence, but not bladder CSM [44]. Patients with CIS only at RC (12\%) had no LN metastasis with durable control even in the case of failure after intravesical therapy [43]. The concomitant CIS was found to be an independent predictor of upper urinary tract carcinoma [45] Conversely in two other studies, concomitant CIS was not independently associated with survival outcomes [46, 47]. A recent analysis of 1128 patients undergoing RC without NAC evaluated the impact of CIS on survival outcomes [29]. The presence of CIS was independently associated with CSM only in the subcohort of patients harboring an organ-confined disease. Patients with concomitant CIS had a significantly increased risk of developing urothelial recurrences regardless of the tumor stage [29]. The most important studies of this paragraph are summarized in Supplementary Table 2.

\section{Pathologic patterns, tumor and node stage}

Positive STSMs seem to be significantly higher in patients with a non-organ-confined disease compared to $\leq$ pT 2 cases 
164

World Journal of Urology (2018) 36:157-170

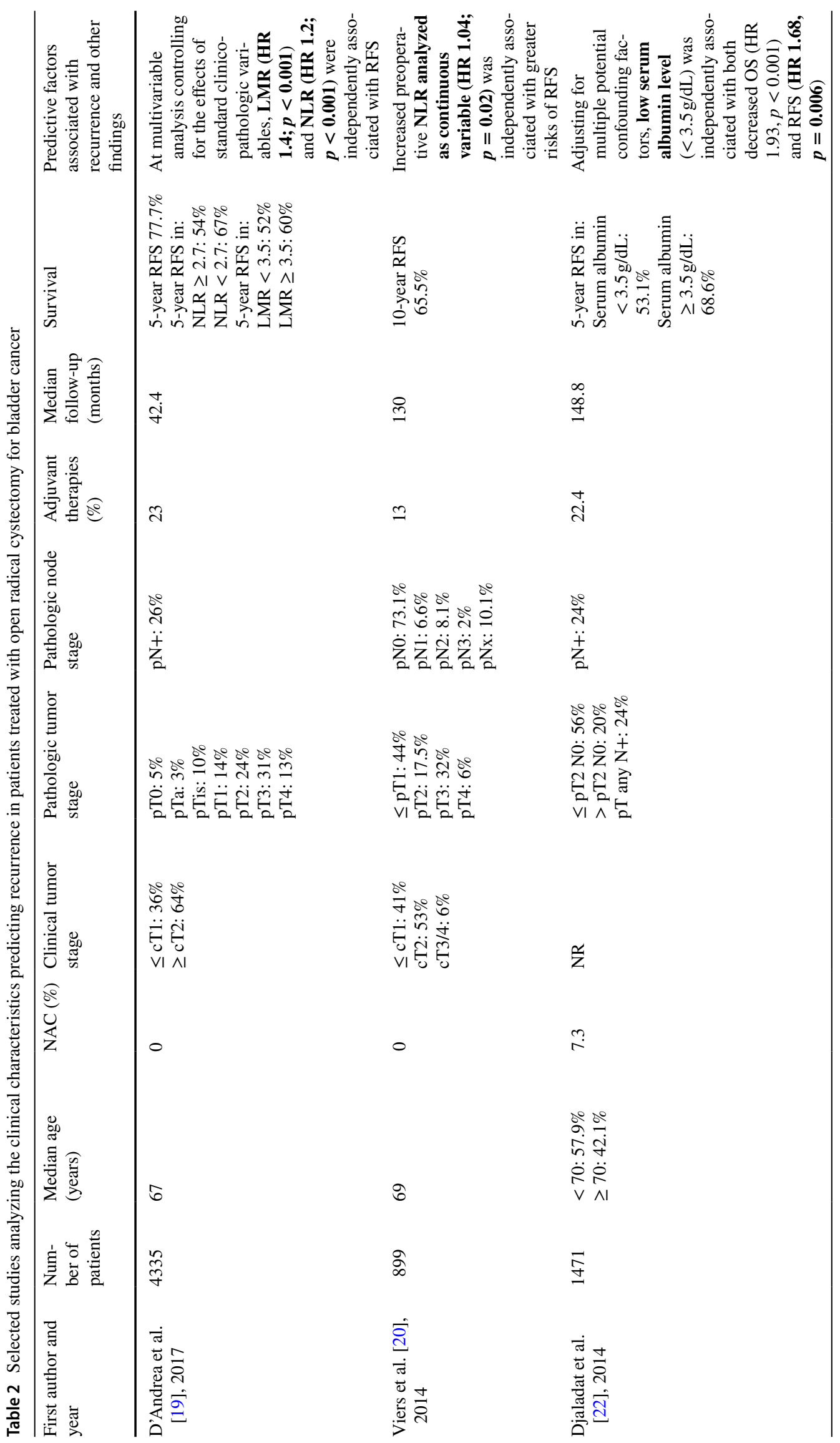

Springer 


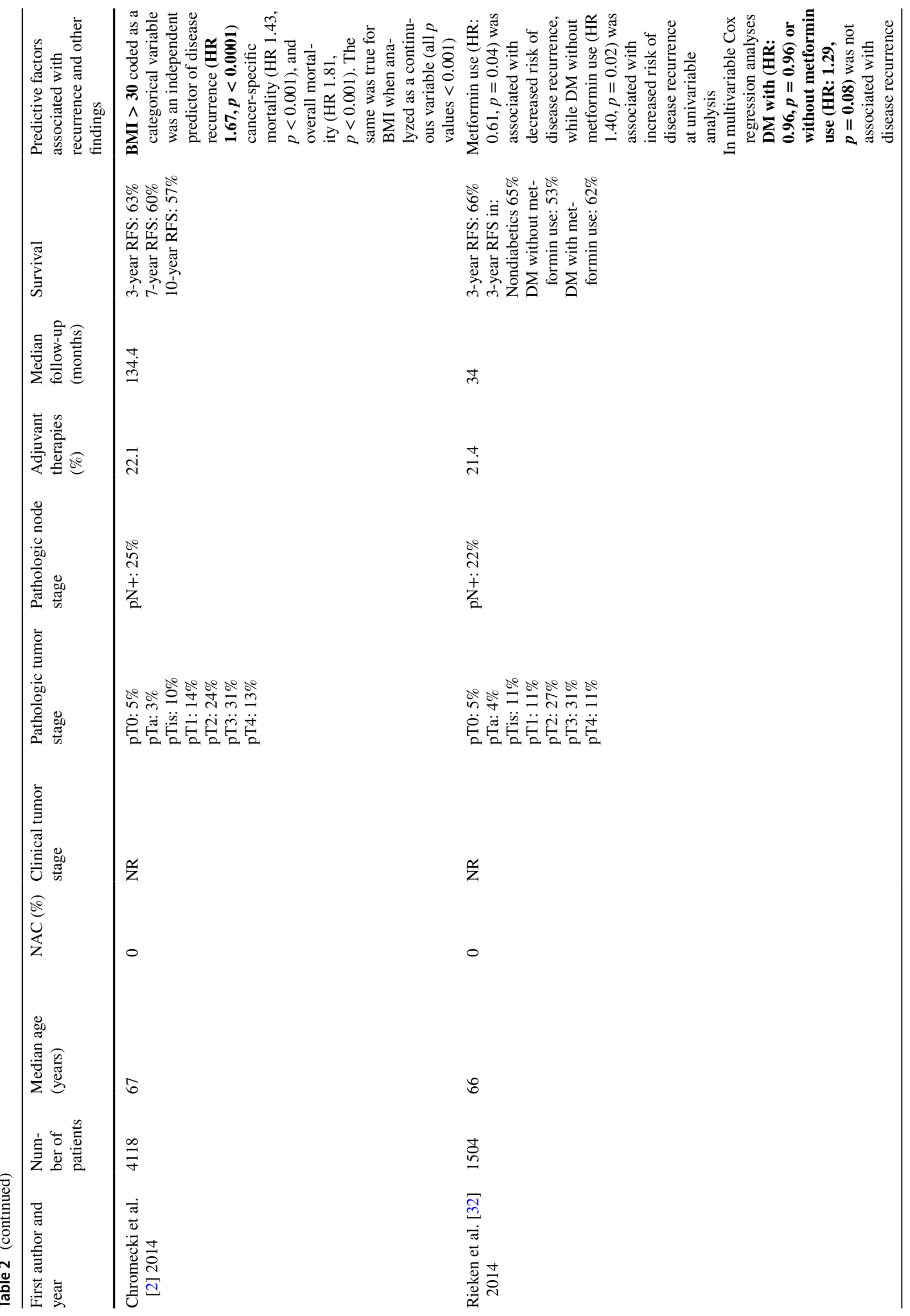




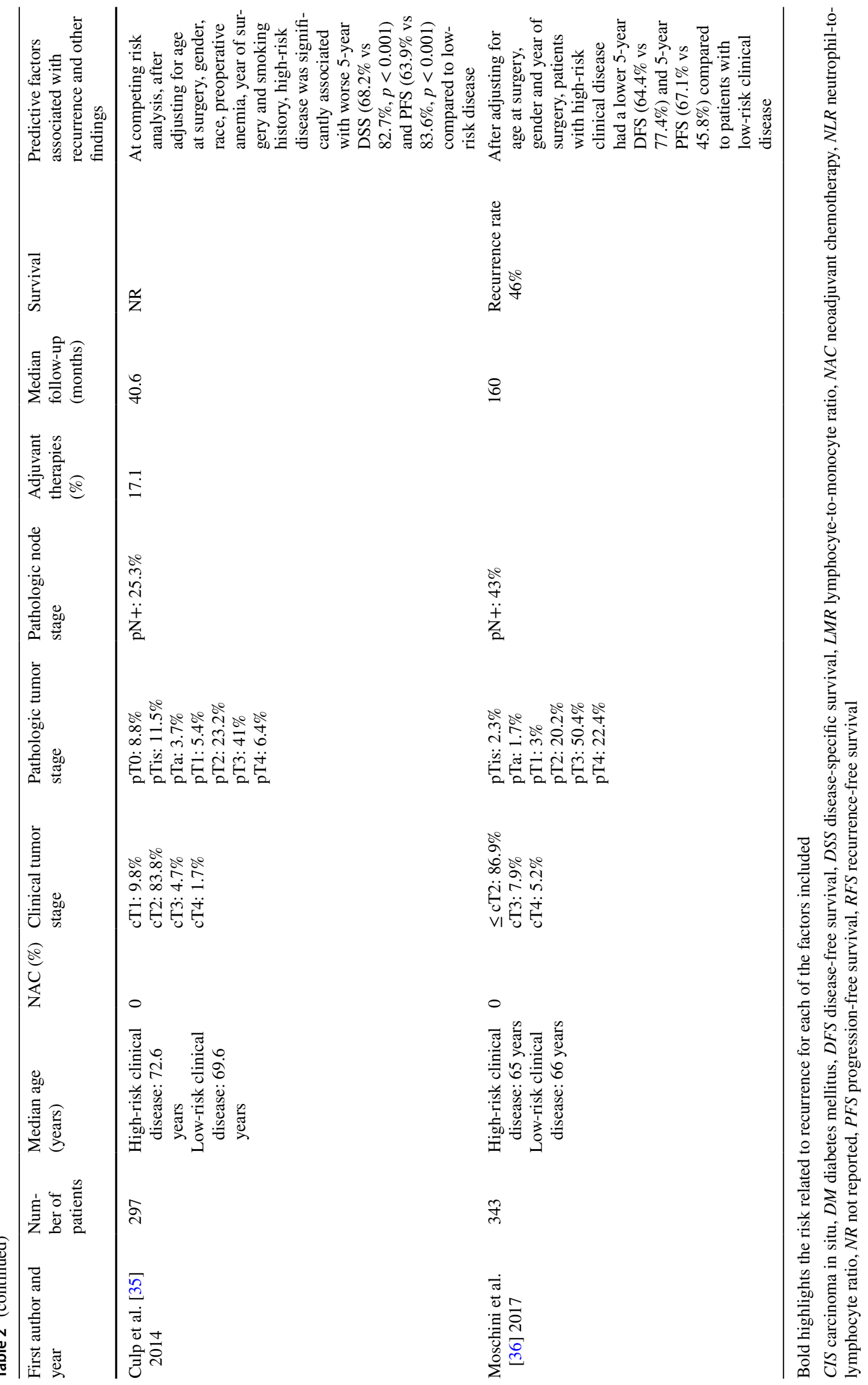


despite the surgical volume of the center $[48,49]$. In a retrospective single-center study evaluating oncologic outcomes of 1589 patients treated with open RC, Dotan et al. showed that the most common locations were the posterior and lateral walls of the bladder and the periprostatic soft tissue [49]. RFS was significantly higher in patients with positive compared to those with negative STSMs. The positive STSMs were independently associated with disease-specific death, but not with metastatic progression after adjusting for clinicopathological factors [49]. Patients with urethral positive surgical margin had an 18-fold higher risk of urethral recurrence. In a multicentre overview of 154 patients with positive STSMs after RC, the local RFS and CSM was significantly lower in patients with positive urethral surgical margin [50]. However, at multivariable analysis the urethral margin was not an independent predictor of CSM.

Tumor and node stage at pathologic evaluation represent two powerful predictors of recurrence and CSM [1]. The International Bladder Cancer Nomogram Consortium analyzed the oncologic outcomes of 9064 patients treated with $\mathrm{RC}$ and lymphadenectomy, including 1550 patients with LN involvement [51]. The Authors developed a postoperative nomogram to predict the risk of recurrence. The pathologic tumor stage and grade and node status were found to have a direct correlation with the risk of recurrence. This association was confirmed in other nomograms developed in wide international series [52, 53].

In a single-center retrospective study enrolling 1388 patients, Umbreit et al. evaluated a multi-factorial, site-specific recurrence model in patients treated with open RC [54]. The abdominal/pelvic, upper urinary tract (UUT), thoracic and bone recurrence rate was $4.8,28,10.3$ and $11 \%$, respectively. After adjusting for clinicopathological features, pT4 stage, positive ureteral margins and multifocality were found to be independent predictors of upper tract recurrence. pT3 and pT4 stage, lymph node invasion and multifocality were found to be independent predictors of abdominal/pelvic, thoracic and bone recurrence.

In a retrospective study involving 1600 patients treated with open RC at a tertiary referral center, extravesical tumor extension and LN density (ratio positive/total node at pathology) greater than $4 \%$ were significantly associated with a higher recurrence rate [55]. Conversely, Tarin et al. analyzed the oncologic outcomes of 591 patients treated with open RC and PLND [56]. After adjusting for perioperative features, the number of positive LN (none, 1 , or 2 or more) was significantly associated with CSM. LN density was not a significant predictor of recurrence or CSS. RFS or CSS did not differ significantly between $\mathrm{pN} 1, \mathrm{pN} 2$, and $\mathrm{pN} 3$. Simone et al. [57] retrospectively identified the $\mathrm{LN}$ density cut-off values to predict CSS in $156 \mathrm{pN}+$ patients treated with $\mathrm{RC}$ and PLND. The thresholds were 11 and $30 \%$ for LN density,
9 and 30 for LN count. In the present study, PLND was the greatest predictor of CSM.

Contrasting studies were reported regarding the influence of PLND extension on LN stage [57, 58]. However, the standard field of PLND should include the lymphatic tissue around the common iliac, external iliac, internal iliac artery, and obturator region. As crossover lymphatic drainage is very common, PLND should always be performed bilaterally [1].

Several studies hypothesized that an extended PLND (ePLND) up to the aortic bifurcation or even super-extended PLND (sePLND) up to the inferior mesenteric artery could be associated with better survival. May et al. [59] showed that the tumor-specific survival and the disease-free interval were improved when an ePLND with equal to or more than 16 removed lymph nodes was performed. Zehnder et al. [60] conducted a retrospective bi-center cohort study on patients who underwent open RC and PLND with curative intent for pT2-3cN0M0 BC. In one institution, all patients were treated with an ePLND up to the mid-upper third of the common iliac vessels. In the other institution, all patients were treated with a sePLND. At multivariable analysis, after adjusting for Institution, pathological subgroup and $\mathrm{AC}, \mathrm{LN}$ status and number of positive LNs were independent risk factors for RFS. The authors concluded that meticulous ePLND appears to provide survival and recurrence outcomes similar to those of sePLND [60]. However, no evidence is reported regarding the possible oncologic benefits of sePLND in particular classes of patients such as those with a clinically suspicious $\mathrm{LN}$ involvement. The most important studies of this paragraph are summarized in Supplementary Table 3.

\section{Conclusions}

A significant proportion of patients relapse after RC for MIBC. Local recurrence rate ranges between 30 and 54\%. Distant recurrence is not often standardized and is reported in up to $50 \%$ of cases.

Higher age, female gender, and smoking exposure at the time of $\mathrm{RC}$ are the most relevant epidemiological predictors of recurrence after RC. Preoperative higher NLR and lower hemoglobin are significantly associated with a worse RFS. Obesity is an independent predictor of RFS. The presence of micropapillary, neuroendocrine features and LVI at TUR specimen, hydroureteronephrosis and cT3b-T4a disease are clinical factors defining a high-risk disease, and are independent predictors of RFS after RC. Among the histologic patterns, LVI, pure VH and concomitant CIS have an independent correlation with RFS after open RC. Advanced tumor stage, multifocality, positive STSMs and $\mathrm{LN}$ involvement are the most relevant pathologic predictors of RFS after RC. A limited PLND resulted to be significant intraoperative predictors of recurrence. Better oncologic 
outcomes were reported in patients submitted to ePLND, while a sePLND seemed to not provide a significantly lower recurrence rate.

Acknowledgements Open access funding provided by Medical University of Vienna.

Author contributions A Mari: Project development, data analysis, manuscript writing. R Campi: Data management, data analysis, manuscript writing. R Tellini: Data collection, data analysis, manuscript writing. G Gandaglia: Data collection, manuscript editing. S Albisinni: Project development, data management, manuscript editing. M Abufaraj: Data management, manuscript editing. G Hatzichristodoulou: Project development, data management, manuscript editing. F Montorsi: Project development, data analysis, manuscript editing. $\mathrm{R}$ van Velthoven: Project development, data analysis, manuscript editing. M Carini: Project development, data analysis, manuscript editing. A Minervini: Project development, data management, manuscript editing. SF Shariat: Project development, data management, data analysis, manuscript writing.

\section{Compliance with ethical standards}

Conflict of interest The authors declare that they have no conflict of interest.

Human participants and/or animals The present research does not involve any human participants or animals.

Informed consent Informed consent was not obtained, as no individual has been included in the study.

Open Access This article is distributed under the terms of the Creative Commons Attribution 4.0 International License (http://creativecommons.org/licenses/by/4.0/), which permits unrestricted use, distribution, and reproduction in any medium, provided you give appropriate credit to the original author(s) and the source, provide a link to the Creative Commons license, and indicate if changes were made.

\section{References}

1. Alfred Witjes J, Lebret T, Comperat EM et al (2016) Updated 2016 EAU guidelines on muscle-invasive and metastatic bladder cancer. Eur Urol. https://doi.org/10.1016/j.eururo.2016.06.020

2. Chromecki TF, Cha EK, Fajkovic H et al (2013) Obesity is associated with worse oncological outcomes in patients treated with radical cystectomy. BJU Int 111:249-255. https://doi. org/10.1111/j.1464-410X.2012.11322.x

3. Xylinas E, Cha EK, Sun M et al (2012) Risk stratification of pT1$3 \mathrm{~N} 0$ patients after radical cystectomy for adjuvant chemotherapy counselling. Br J Cancer 107:1826-1832. https://doi.org/10.1038/ bjc. 2012.464

4. Zehnder P, Studer UE, Skinner EC et al (2013) Unaltered oncological outcomes of radical cystectomy with extended lymphadenectomy over three decades. BJU Int 112:E51-E58. https://doi. org/10.1111/bju.12215

5. Roupret M, Drouin SJ, Larre S et al (2011) Oncologic outcomes and survival in pT0 tumors after radical cystectomy in patients without neoadjuvant chemotherapy: results from a large multicentre collaborative study. Ann Surg Oncol 18:3833-3838. https:// doi.org/10.1245/s10434-011-1824-y
6. Deeks JJ, Dinnes J, D'Amico R, et al (2003) Evaluating nonrandomised intervention studies. Health Technol Assess 7:iii-X, $1-173$

7. Griffiths G, Hall R, Sylvester R et al (2011) International phase III trial assessing neoadjuvant cisplatin, methotrexate, and vinblastine chemotherapy for muscle-invasive bladder cancer: longterm results of the BA06 30894 trial. J Clin Oncol 29:2171-2177. https://doi.org/10.1200/JCO.2010.32.3139

8. Grossman HB, Natale RB, Tangen CM et al (2003) Neoadjuvant chemotherapy plus cystectomy compared with cystectomy alone for locally advanced bladder cancer. N Engl J Med 349:859-866. https://doi.org/10.1056/NEJMoa022148

9. Tracey E, Roder D, Luke C, Bishop J (2009) Bladder cancer survivals in New South Wales, Australia: why do women have poorer survival than men? BJU Int 104:498-504. https://doi. org/10.1111/j.1464-410X.2009.08527.x

10. Mungan NA, Kiemeney LA, van Dijck JA et al (2000) Gender differences in stage distribution of bladder cancer. Urology 55:368-371

11. Messer JC, Shariat SF, Dinney CP et al (2014) Female gender is associated with a worse survival after radical cystectomy for urothelial carcinoma of the bladder: a competing risk analysis. Urology 83:863-867. https://doi.org/10.1016/j. urology.2013.10.060

12. Mitra AP, Skinner EC, Schuckman AK et al (2014) Effect of gender on outcomes following radical cystectomy for urothelial carcinoma of the bladder: a critical analysis of 1994 patients. Urol Oncol Semin Orig Invest 32:52.e1-52.e9. https://doi. org/10.1016/j.urolonc.2013.08.007

13. Abdollah F, Sun M, Schmitges J et al (2012) Development and validation of a reference table for prediction of postoperative mortality rate in patients treated with radical cystectomy: a population-based study. Ann Surg Oncol 19:309-317. https:// doi.org/10.1245/s10434-011-1852-7

14. Horovitz D, Turker P, Bostrom PJ et al (2012) Does patient age affect survival after radical cystectomy? BJU Int 110:E486E493. https://doi.org/10.1111/j.1464-410X.2012.11180.x

15. Nielsen ME, Shariat SF, Karakiewicz PI et al (2007) Advanced age is associated with poorer bladder cancer-specific survival in patients treated with radical cystectomy. Eur Urol 51:698-699. https://doi.org/10.1016/j.eururo.2006.11.004

16. Rink M, Zabor EC, Furberg H et al (2013) Impact of smoking and smoking cessation on outcomes in bladder cancer patients treated with radical cystectomy. Eur Urol 64:456-464. https:// doi.org/10.1016/j.eururo.2012.11.039

17. Bostrom PJ, Alkhateeb S, Trottier G et al (2012) Sex differences in bladder cancer outcomes among smokers with advanced bladder cancer. BJU Int 109:70-76. https://doi. org/10.1111/j.1464-410X.2011.10371.x

18. Lee C, Kim KH, You D et al (2012) Smoking and survival after radical cystectomy for bladder cancer. Urology 80:1307-1312. https://doi.org/10.1016/j.urology.2012.08.026

19. D'Andrea D, Moschini M, Gust KM et al (2017) Lymphocyteto-monocyte ratio and neutrophil-to-lymphocyte ratio as biomarkers for predicting lymph node metastasis and survival in patients treated with radical cystectomy. J Surg Oncol 115:455461. https://doi.org/10.1002/jso.24521

20. Viers BR, Boorjian SA, Frank I et al (2014) Pretreatment neutrophil-to-lymphocyte ratio is associated with advanced pathologic tumor stage and increased cancer-specific mortality among patients with urothelial carcinoma of the bladder undergoing radical cystectomy. Eur Urol 66:1157-1164. https://doi. org/10.1016/j.eururo.2014.02.042

21. Temraz S, Mukherji D, Farhat ZAA et al (2014) Preoperative lymphocyte-to-monocyte ratio predicts clinical outcome in patients undergoing radical cystectomy for transitional cell 
carcinoma of the bladder: a retrospective analysis. BMC Urol 14:76. https://doi.org/10.1186/1471-2490-14-76

22. Djaladat H, Bruins HM, Miranda G et al (2014) The association of preoperative serum albumin level and American Society of Anesthesiologists (ASA) score on early complications and survival of patients undergoing radical cystectomy for urothelial bladder cancer. BJU Int 113:887-893. https://doi.org/10.1111/ bju. 12240

23. Liu J, Dai Y, Zhou F et al (2016) The prognostic role of preoperative serum albumin/globulin ratio in patients with bladder urothelial carcinoma undergoing radical cystectomy. Urol Oncol 34:484.e1-484.e8. https://doi.org/10.1016/j.urolonc.2016.05.024

24. Moschini M, Suardi N, Pellucchi F et al (2014) Impact of preoperative thrombocytosis on pathological outcomes and survival in patients treated with radical cystectomy for bladder carcinoma. Anticancer Res 34:3225-3230

25. Xia L, Guzzo TJ (2017) Preoperative anemia and low hemoglobin level are associated with worse clinical outcomes in patients with bladder cancer undergoing radical cystectomy: a meta-analysis. Clin Genitourin Cancer 15(263-272):e4. https://doi.org/10.1016/j. clgc.2016.08.017

26. Engel O, Soave A, Peine $\mathrm{S}$ et al (2015) The impact of the AB0 and the Rhesus blood group system on outcomes in bladder cancer patients treated with radical cystectomy. World J Urol 33:17691776. https://doi.org/10.1007/s00345-015-1531-6

27. Miyake M, Morizawa Y, Hori S et al (2017) Integrative assessment of pretreatment inflammation-, nutrition-, and muscle-based prognostic markers in patients with muscle-invasive bladder cancer undergoing radical cystectomy. Oncology 93:259-269. https:// doi.org/10.1159/000477405

28. Koppie TM, Serio AM, Vickers AJ et al (2008) Age-adjusted Charlson comorbidity score is associated with treatment decisions and clinical outcomes for patients undergoing radical cystectomy for bladder cancer. Cancer 112:2384-2392. https://doi. org/10.1002/cncr.23462

29. Moschini M, Shariat SF, Abufaraj M et al (2017) The presence of carcinoma in situ at radical cystectomy increases the risk of urothelial recurrence: Implications for follow-up schemes. Urol Oncol 35:151.e17-151.e23. https://doi.org/10.1016/j. urolonc.2016.11.003

30. Mayr R, May M, Burger M et al (2014) The Charlson comorbidity index predicts survival after disease recurrence in patients following radical cystectomy for urothelial carcinoma of the bladder. Urol Int 93:303-310. https://doi.org/10.1159/000362421

31. Dabi Y, Rouscoff Y, Anract J et al (2017) Impact of body mass index on the oncological outcomes of patients treated with radical cystectomy for muscle-invasive bladder cancer. World J Urol 35:229-235. https://doi.org/10.1007/s00345-016-1852-0

32. Rieken M, Xylinas E, Kluth L et al (2014) Effect of diabetes mellitus and metformin use on oncologic outcomes of patients treated with radical cystectomy for urothelial carcinoma. Urol Oncol 32:49.e7-14. https://doi.org/10.1016/j.urolonc.2013.07.006

33. Milowsky MI, Rumble RB, Booth CM et al (2016) Guideline on muscle-invasive and metastatic bladder cancer (European Association of Urology Guideline): American society of clinical oncology clinical practice guideline endorsement. J Clin Oncol 34:1945-1952. https://doi.org/10.1200/JCO.2015.65.9797

34. Hollenbeck BK, Miller DC, Dunn RL et al (2005) The effects of stage divergence on survival after radical cystectomy for urothelial cancer. Urol Oncol 23:77-81. https://doi.org/10.1016/j. urolonc.2004.08.012

35. Culp SH, Dickstein RJ, Grossman HB et al (2014) Refining patient selection for neoadjuvant chemotherapy before radical cystectomy. J Urol 191:40-47. https://doi.org/10.1016/j.juro.2013.07.061

36. Moschini M, Soria F, Klatte T et al (2017) Validation of preoperative risk grouping of the selection of patients most likely to benefit from neoadjuvant chemotherapy before radical cystectomy. Clin Genitourin Cancer 15:e267-e273. https://doi.org/10.1016/j. clgc.2016.07.014

37. Lotan Y, Gupta A, Shariat SF et al (2005) Lymphovascular invasion is independently associated with overall survival, causespecific survival, and local and distant recurrence in patients with negative lymph nodes at radical cystectomy. J Clin Oncol 23:6533-6539. https://doi.org/10.1200/JCO.2005.05.516

38. Xylinas E, Rink M, Robinson BD et al (2013) Impact of histological variants on oncological outcomes of patients with urothelial carcinoma of the bladder treated with radical cystectomy. Eur J Cancer 49:1889-1897. https://doi.org/10.1016/j.ejca.2013.02.001

39. Soave A, Schmidt S, Dahlem R et al (2015) Does the extent of variant histology affect oncological outcomes in patients with urothelial carcinoma of the bladder treated with radical cystectomy? Urol Oncol 33:21.e1-9. https://doi.org/10.1016/j. urolonc.2014.10.013

40. Fairey AS, Daneshmand S, Wang L et al (2014) Impact of micropapillary urothelial carcinoma variant histology on survival after radical cystectomy. Urol Oncol 32:110-116. https://doi. org/10.1016/j.urolonc.2012.04.020

41. Moschini M, Shariat SF, Luciano R et al (2016) Pure but not mixed histologic variants are associated with poor survival at radical cystectomy in bladder cancer patients. Clin Genitourin Cancer. https://doi.org/10.1016/j.clgc.2016.12.006

42. Zargar-Shoshtari K, Sverrisson EF, Sharma P et al (2016) Clinical outcomes after neoadjuvant chemotherapy and radical cystectomy in the presence of urothelial carcinoma of the bladder with squamous or glandular differentiation. Clin Genitourin Cancer 14:82-88. https://doi.org/10.1016/j.clgc.2015.08.006

43. Shariat SF, Palapattu GS, Amiel GE et al (2006) Characteristics and outcomes of patients with carcinoma in situ only at radical cystectomy. Urology 68:538-542. https://doi.org/10.1016/j. urology.2006.03.033

44. Shariat SF, Palapattu GS, Karakiewicz PI et al (2007) Concomitant carcinoma in situ is a feature of aggressive disease in patients with organ-confined TCC at radical cystectomy. Eur Urol 51:152160. https://doi.org/10.1016/j.eururo.2006.08.037

45. Wheat JC, Weizer AZ, Wolf JSJ et al (2012) Concomitant carcinoma in situ is a feature of aggressive disease in patients with organ confined urothelial carcinoma following radical nephroureterectomy. Urol Oncol 30:252-258. https://doi.org/10.1016/j. urolonc.2010.01.001

46. Nuhn P, Bastian PJ, Novara G et al (2011) Concomitant carcinoma in situ in cystectomy specimens is not associated with clinical outcomes after surgery. Urol Int 87:42-48. https://doi. org/10.1159/000325463

47. Yafi FA, Aprikian AG, Chin JL et al (2014) Impact of concomitant carcinoma in situ on upstaging and outcome following radical cystectomy for bladder cancer. World J Urol 32:1295-1301. https:// doi.org/10.1007/s00345-013-1207-z

48. Herr H, Lee C, Chang S, Lerner S (2004) Standardization of radical cystectomy and pelvic lymph node dissection for bladder cancer: a collaborative group report. J Urol 171:1823-1828. https:// doi.org/10.1097/01.ju.0000120289.78049.0e

49. Dotan ZA, Kavanagh K, Yossepowitch O et al (2007) Positive surgical margins in soft tissue following radical cystectomy for bladder cancer and cancer specific survival. J Urol 178:2308-2313. https://doi.org/10.1016/j.juro.2007.08.023

50. Neuzillet Y, Soulie M, Larre S et al (2013) Positive surgical margins and their locations in specimens are adverse prognosis features after radical cystectomy in non-metastatic carcinoma invading bladder muscle: results from a nationwide case-control study. BJU Int 111:1253-1260. https://doi. org/10.1111/j.1464-410X.2012.11664.X 
51. Bochner BH, Kattan MW, Vora KC (2006) Postoperative nomogram predicting risk of recurrence after radical cystectomy for bladder cancer. J Clin Oncol 24:3967-3972. https://doi. org/10.1200/JCO.2005.05.3884

52. Simone G, Bianchi M, Giannarelli D et al (2015) Development and external validation of nomograms predicting disease-free and cancer-specific survival after radical cystectomy. World J Urol 33:1419-1428. https://doi.org/10.1007/s00345-014-1465-4

53. Di Trapani E, Sanchez-Salas R, Gandaglia G et al (2016) A nomogram predicting the cancer-specific mortality in patients eligible for radical cystectomy evaluating clinical data and neoadjuvant cisplatinum-based chemotherapy. World J Urol 34:207-213. https://doi.org/10.1007/s00345-015-1640-2

54. Umbreit EC, Crispen PL, Shimko MS et al (2010) Multifactorial, site-specific recurrence model after radical cystectomy for urothelial carcinoma. Cancer 116:3399-3407. https://doi.org/10.1002/ cncr.25202

55. Bruins HM, Huang GJ, Cai J et al (2009) Clinical outcomes and recurrence predictors of lymph node positive urothelial cancer after cystectomy. J Urol 182:2182-2187. https://doi.org/10.1016/j. juro.2009.07.017

56. Tarin TV, Power NE, Ehdaie B et al (2012) Lymph node-positive bladder cancer treated with radical cystectomy and lymphadenectomy: effect of the level of node positivity. Eur Urol 61:10251030. https://doi.org/10.1016/j.eururo.2012.01.049
57. Simone G, Papalia R, Ferriero M et al (2012) Development and external validation of lymph node density cut-off points in prospective series of radical cystectomy and pelvic lymph node dissection. Int J Urol 19:1068-1074. https://doi. org/10.1111/j.1442-2042.2012.03103.x

58. Abdi H, Pourmalek F, Gleave ME et al (2016) Balancing risk and benefit of extended pelvic lymph node dissection in patients undergoing radical cystectomy. World J Urol 34:41-48. https:// doi.org/10.1007/s00345-015-1734-x

59. May M, Herrmann E, Bolenz C et al (2011) Association between the number of dissected lymph nodes during pelvic lymphadenectomy and cancer-specific survival in patients with lymph node-negative urothelial carcinoma of the bladder undergoing radical cystectomy. Ann Surg Oncol 18:2018-2025. https://doi. org/10.1245/s10434-010-1538-6

60. Zehnder P, Studer UE, Skinner EC et al (2011) Super extended versus extended pelvic lymph node dissection in patients undergoing radical cystectomy for bladder cancer: a comparative study. J Urol 186:1261-1268. https://doi.org/10.1016/j.juro.2011.06.004 